Horizons philosophiques

\title{
Considérations éthiques sur le congé en milieu hospitalier de
} courte durée

\author{
Alberte Perron, Robert Clément, David J. Roy, Lise Sauvé-Henry et Thérèse \\ Leroux
}

Volume 4, numéro 2, printemps 1994

Médecines impossibles?

URI : https://id.erudit.org/iderudit/800952ar

DOI : https://doi.org/10.7202/800952ar

Aller au sommaire du numéro

Éditeur(s)

Collège Édouard-Montpetit

ISSN

1181-9227 (imprimé)

1920-2954 (numérique)

Découvrir la revue

Citer cet article

Perron, A., Clément, R., Roy, D. J., Sauvé-Henry, L. \& Leroux, T. (1994).

Considérations éthiques sur le congé en milieu hospitalier de courte durée.

Horizons philosophiques, 4(2), 113-120. https://doi.org/10.7202/800952ar 


\section{CONSIDÉRATIONS ÉTHIQUES SUR LE CONGÉ EN MILIEU HOSPITALIER DE COURTE DURÉE}

Un des mandats dévolus au service social hospitalier s'inscrit dans le cadre de la participation au plan de congé du patient II remonte au début du siècle, depuis que les services sociaux hospitaliers existent. C'est une préoccupation primordiale mais peu souvent évoquée, sauf en situation de crise.

Pourquoi le plan de congé? Les personnes qui travaillent en milieu hospitalier savent que le service social est impliqué dans le plan de congé du patient. Pas dans tous les plans de congé toutefois, mais bien ceux qui pourraient être préjudiciables au patient et/ou à sa famille, pour des raisons d'ordre social, médical, économique, de qualité de vie, ou pour cause d'incapacité du milieu et des ressources à fournir au patient les soins adéquats.

Nous savons aussi que dans la situation complexe et difficile de la planification d'un départ, nous pouvons être confrontés aux attentes et aux désirs du patient et de sa famille, aux conflits familiaux, à des questions de gestion des lits, aux ressources limitées, aux systèmes longs et complexes de placement, etc. Nous pouvons ainsi facilement nous retrouver en situation de crise, confrontés à des problèmes éthiques : conflits de valeurs, de croyances, etc.

À partir des histoires de cas qui concernent notre centre hospitalier, nous avons retenu trois cas représentatifs de la population qui nous est référée. La première situation que nous exposons est celle d'un homme de 75 ans, hospitalisé pour des problèmes circulatoires. Ayant déjà été amputé d'une jambe quelques années auparavant, il a dû subir l'amputation de l'autre jambe au cours de la présente hospitalisation.

Monsieur D. est marié et vit avec son épouse à une centaine de kilomètres de Montréal. II a six enfants, tous mariés et 
quelques petits-enfants, dont plusieurs demeurent à Montréal. Après avoir reçu les services de réadaptation nécessaires, il redevient autonome dans toutes ses A.V.Q. (activités de la vie quotidienne), avec l'aide de prothèses et d'une chaise roulante. Monsieur D. est une force de la nature; il est très costaud et paraît la cinquantaine.

Or, Monsieur D. refuse de retourner chez lui en prétextant qu'il ne peut vivre avec son épouse, qu'il se sentirait diminué devant elle et qu'il serait rejeté par elle. II serait isolé, loin des services auxquels il a droit et il veut être placé à Montréal à tout prix.

Des démarches de placement sont entreprises. Monsieur D. est refusé en centre d'accueil comme en H.L.M. et lui-même décline les ressources privées et une famille d'accueil de type «appartement adapté».

Les péripéties durent deux ans. Les motifs se multiplient pour récuser toute aide : premier refus de signer les documents pour le placement, puis acceptation pour la signature quelques semaines plus tard, rejet ultérieur de la ressource proposée et nouvelle orientation; il refuse alors toute autre démarche car sa prothèse est mal ajustée, il profère des injures, etc. La roue tourne ainsi jusqu'au point où Monsieur D. évolue sur le terrain de l'hôpital en chaise roulante, les moignons à découvert, avec une pancarte précisant qu'il est mal soigné.

L'épouse et les enfants refusent de participer à quelque plan que ce soit, à l'exception qu'une des filles expliquent que son père a toujours eu un sale caractère, que sa mère "décompensera" s'il retourne avec elle, et qu'ils ont tous eu une vie très difficile avec lui et par conséquent plus il sera loin, mieux ça vaudra.

Après deux ans, il y a réunion de tous les intervenants impliqués et le congé est donné. Monsieur $D$. réagit en disant : "Si c'est comme ça, je retourne chez moi», tout en lançant des injures à l'adresse de tous les intervenants. Nous n'en avons plus jamais entendu parler. II retourna chez lui, dans une 
maison entièrement adaptée pour handicapé. Cette dernière information, personne, ni même le C.L.S.C. du coin qui le savait, ne nous l'avait transmise, sous prétexte de protéger l'épouse, qui paraît-il était psychologiquement fragile, et voulait se séparer de son mari en conservant le domicile conjugal.

Notre deuxième cas est celui d'un jeune homme d'une vingtaine d'années, souffrant de céphalées intenses, prises d'abord pour des migraines et traitées par de l'aspirine. II fut admis à l'urgence pour une hémorragie intracérébrale brutale. Après examen, on doit pratiquer d'urgence une craniotomie; mais vingt-quatre heures après l'intervention, l'état du patient s'aggrave brutalement et évolue vers un coma aréactif. II est réopéré d'urgence, avec pour résultat une légère amélioration en phase post-opératoire. Le patient reste plusieurs mois aux soins intensifs. Malgré une légère amélioration régulière, il reste toutefois dans un état de tétraparésie accompagné de multiples contractions, qu'on essaie de diminuer en recourant à la physiothérapie.

Après cinq mois, les spécialistes pensent que le patient a atteint un état chronique et qu'il peut être transféré en soins prolongés sans danger supplémentaire. Le médecin en avise la famille.

Très unie, celle-ci est composée des parents du patient, fils aîné, de deux sœurs et de la conjointe du patient. La mère travaille ainsi que la conjointe, et les dèux sœurs sont aux études. Le père est invalide et souffre de problèmes cardiaques. Il décédera d'ailleurs d'un infarctus à la suite d'une première visite à son fils.

Les membres de la famille - la mère et la conjointe du patient surtout - unissent leurs efforts et consacrent tout leur temps disponible à l'amélioration de l'état du malade. Elles contrôlent l'ensemble des soins qui lui sont prodigués et donnent elles-mêmes des soins.

Mais la mère et la conjointe refusent le verdict de chronicité et, par le fait même, le transfert en soins prolongés, qu'elles 
perçoivent comme un abandon. De plus, elles font porter sur l'hôpital le fardeau de la responsabilité de l'état actuel du patient.

Elles exigent plutôt un transfert vers un centre de réadaptation, ce qui est effectué par le physiatre tout en sachant que les progrès resteront limités.

Après quelques mois, la mère et la conjointe rencontrent les membres de l'équipe soignante du centre de réadaptation pour faire le point. On les informe que tout a été tenté pour obtenir les meilleurs résultats et que le patient ne peut que bénéficier de soins de confort. II devrait donc revenir à l'Hôtel-Dieu de Montréal.

Conjointement avec l'infirmière de cethôpital, la travailleuse sociale convainc la mère et la conjointe de préparer le dossier en vue d'un placement en soins prolongés. La famille se résigne à cette démarche, en la considérant comme une étape en attendant la création d'un centre spécialisé pour les patients victimes de traumatisme craniocérébral. Par ailleurs, la mère et la conjointe sont des membres actives de l'Association québécoise des traumatisés crâniens.

Dans l'attente du transfert, la famille continue à se relayer auprès du patient pour lui apporter des soins. Le malade est à son domicile en fin de semaine, et il suit des sessions privées de physiothérapie dans un autre centre de réadaptation.

Au moment du transfert, la famille rejette l'établissement proposé et exige un autre centre de son choix, ce qui prolonge encore le séjour du patient à l'hôpital. Finalement, au niveau du système régional de placement, on accepte un compromis et le patient quitte l'hôpital après deux ans et demi, pour entrer dans le centre de soins prolongés choisi par la famille.

La troisième situation est illustrée parune dame, âgée de 75 ans, en mauvaise santé. Elle entre à l'hôpital pour un problème gastrique qui l'affaiblit beaucoup. Cette affection est traitée, mais à cause d'un A.C.V. (anévrisme cardio-vasculaire) antérieur, on juge qu'elle n'est pas suffisamment fonctionnelle pour réintégrer son domicile où elle vit seule. Elle a un fils qui 
travaille, lui rend visite de temps à autre et s'occupe de ses affaires.

Tout en gardant l'hébergement comme option ultime, on transfère la patiente dans un centre de réadaptation, en vue d'améliorer ses capacités fonctionnelles. Cet objectif atteint au niveau de la réadaptation, on la renvoie à l'Hôtel-Dieu pour une nouvelle investigation, tout en confirmant le besoin d'hébergement.

À l'Hôtel-Dieu, la patiente est référée au service social en vue d'un placement. La travailleuse sociale rencontre la patiente qui, elle, s'en remet à son fils. A l'issue d'une rencontre, ce dernier rejette l'orientation placement parce que, selon lui, sa mère est toujours en investigation sur le plan médical. La travailleuse sociale discute avec le médecin de l'attitude du fils, et elle communique également avec l'infirmière au sujet de l'état de la patiente.

De son côté, le fils entre régulièrement en contact avec les médecins pour les informer des malaises dont se plaint sa mère et pour demander de nouveaux examens. La situation se prolonge ainsi pendant plusieurs mois, les communications s'effectuant parallèlement entre les différents intervenants, la mère et son fils.

Pendant ce temps, les consultations se poursuivent même après que le médecin et la travailleuse sociale, chacun de leur côté, aient rempli la déclaration d'hébergement. Le fils réagit en expédiant une lettre d'avocat à l'administration pour faire annuler cette déclaration.

Les rencontres de la travailleuse sociale avec le fils restent infructueuses; ce dernier met constamment de l'avant les problèmes de santé de sa mère et fait appel au médecin pour réclamer de nouveaux soins.

L'infirmière s'entretient avec la travailleuse sociale au sujet de cette situation en cul-de-sac. La travailleuse sociale suggère alors que l'équipe soignante - médecin, infirmière et travailleuse sociale - rencontre le fils pour clarifier la situation. Cette suggestion est accueillie favorablement par les membres de l'équipe. 
Et ce n'est qu'à la suite de cette rencontre qu'on obtient la collaboration du fils.

\section{RÉFLEXIONS}

À partir de l'analyse de ces trois cas, nous avons tenté de faire ressortir les aspects éthiques du congé. II est utile de rappeler d'abord que tous les acteurs sont en interaction dynamique : le patient se doit d'être au centre de cette dynamique; le travailleur social est celui qui aborde avec le patient la planification du congé et l'orientation future. Outre le travailleur social, les partenaires professionnels à l'intérieur de l'établissement sont le médecin qui a la responsabilité de signer le congé, les infirmières qui dispensent les soins et les autres soignants dont le patient requiert les services, ainsi que l'administration hospitalière. À l'extérieur de l'établissement, d'autres partenaires entrent aussi en interaction avec le patient. II y a d'abord la famille, les proches qui appuient le patient ou, à l'inverse l'ignorent et même le rejettent; ils peuvent aussi tenter de l'exploiter. II y a également le réseau des ressources communautaires qui conditionne les recherches du travailleur social et influence le patient et/ou sa famille. Chaque noyau est dynamique par rapport aux autres et tous le sont par rapport au patient.

Par ailleurs, la Charte Canadienne, la Charte Québécoise, les politiques sociales, le code des professions, les codes de déontologie, etc., proposent un cadre d'action, précisent ce qui doit être fait ou pas, ce qui est permis ou non et différencient ainsi le bien du mal. Une morale de l'agir s'est donc développée et tout intervenant ou soignant doit y adhérer.

Le professionnel doit respecter les droits des individus : choix de l'établissement, choix du professionnel, droit à l'information, respect de l'intégrité, etc. Certes, dans la réalité de tous les jours, les professionnels et les institutions s'orientent en fonction des choix collectifs. Un citoyen peut choisir de se faire traiter à Hôtel-Dieu de Montréal, c'est un droit individuel et légitime. Mais tous les citoyens du Québec ne pourraient choisir de se faire traiter à l'Hôtel-Dieu de Montréal. Nous acceptons le 
fait que le droit individuel d'un citoyen est à la limite du droit individuel d'un autre et est confronté aux limites des organisations, des lois et règlements, des choix de société. L'individu peut exiger le respect de ses droits mais il ne peut outrepasser les limites des organisations, les limites des thérapeutiques disponibles et celles des professionnels en cause.

Si l'équation besoins $=$ désirs $=$ ressources est valable en tout temps et en tout lieu, nous avons une harmonie entre les droits des uns, les droits des autres et les choix de société. Dans le cas contraire, il y a problème, confrontation et il est nécessaire de réfléchir sur notre action professionnelle.

Les choix éthiques sont singuliers et personnalisés. Au préalable, on doit établir un consensus sur les grands principes, ce qui exige de nombreuses reformulations. L'éthique est inductive : chaque cas est examiné à la lumière des principes et débouche sur une norme particulière. C'est l'examen de plusieurs cas qui nous amène à réviser les grands principes moraux. De plus, avec l'éthique, on entre dans le domaine pragmatique de la perception et de la communication. II faut s'orienter vers la discussion plutôt que vers les affrontements; la reconnaissance des personnes comme partenaires de discussion est également essentielle.

L'analyse critique des divers jugements en vue d'une résolution des conflits est une démarche proprement philosophique. L'éthique s'inscrit dans cette analyse critique, en recherchant une cohérence entre connaissance et action.

En conclusion, lorsque nous avons amorcé cette étude, nous avions cru que les problèmes étaient avant tout d'ordre organisationnel et structurel. Nous savons tous que la bureaucratie est lourde, que les cahiers des politiques et procédures sont imposants et qu'il en va de même pour les lois et règlements. Or nous nous sommes vite aperçus que les problèmes étaient avant tout de l'ordre de la perception et de la communication, sans vouloir négliger les autres facteurs.

Si les intervenants en situation difficile (et même les situations moins difficiles) disposaient d'un lieu pour échanger sur 
leurs perceptions des faits, leurs valeurs, leurs croyances, en d'autres mots pour communiquer, ils arriveraient plus facilement à une cohérence dans l'approche et à un plan d'action concerté. Dès lors, les situations de crise seraient réduites au minimum, à la plus grande satisfaction des intervenants, tout en permettant de fournir un meilleur service au patient. Nous croyons qu'il faut abandonner une pensée linéaire pour une pensée circulaire.

Alberte Perron, travailleuse sociale :

Service social, Hôtel-Dieu de Mtl

Robert Clément, travailleur social : Chef du Service social, Hôtel-Dieu de MtI

David J. Roy, bioéthicien : Directeur du centre de bioéthique Institut de recherche clinique de MtI

Lise Sauvé-Henry, travailleuse sociale : Service social, Hôtel-Dieu de Mtl

Thérèse Leroux, avocate : Centre de recherche en droit public Université de Montréal 